\title{
Critical Analysis of Treatment Trials of Rhesus Macaques Infected with Borrelia burgdorferi Reveals Important Flaws in Experimental Design
}

\author{
Gary P. Wormser, ${ }^{1}$ Phillip J. Baker, ${ }^{2}$ Susan O'Connell, ${ }^{3}$ Andrew R. Pachner, ${ }^{4}$ \\ Ira Schwartz, and Eugene D. Shapiro ${ }^{6}$
}

\begin{abstract}
A critical analysis of two treatment trials of Chinese rhesus macaques infected with Borrelia burgdorferi indicates that insufficient attention was placed on documenting the blood levels, pharmacokinetics, and pharmacodynamic parameters of the antibiotics used in this host. Consequently, it is impossible to conclude that the findings have validity in judging the efficacy of doxycycline or ceftriaxone for the treatment of Borrelia burgdorferi in this animal model.
\end{abstract}

Key Words: Antibiotic therapy-C6 antibody—Ceftriaxone-Doxycycline-Erythema migrans-Lyme diseaseNon-human primates- Post-treatment Lyme disease syndrome.

E MBERS AND ASSOCIATES (2012) RECENTLY REPORTED the results of two experiments in which Chinese rhesus macaques infected with Borrelia burgdorferi received either antibiotics or sham therapy (controls). They state that despite "aggressive" antibiotic therapy with ceftriaxone, followed by doxycycline in experiment 1 or doxycycline treatment alone in experiment 2, B. burgdorferi "appears to become tolerant" and "persists." They also state that their results raise important questions "about the pathogenicity of antibiotic-tolerant persisters and whether or not they can contribute to symptoms post-treatment."

None of the non-human primates (NHPs) were described as having objective clinical manifestations of infection (e.g., erythema migrans, neurologic dysfunction, or arthritis), either during or after treatment. Thus resolution of such findings was not used to measure antibiotic efficacy. Obviously, the presence or absence of purely subjective complaints such as fatigue likewise could not be reliably assessed in this system. Instead, the evaluation of antibiotic efficacy was based on a positive result for any one of four different laboratory assays (detection of the growth of B. burgdorferi in culture, identification of borrelial DNA or RNA by poly- merase chain reaction [PCR], or the detection of B. burgdorferi antigen by an immunofluorescence assay [IFA] in histologic sections) in experiment 1 . Xenodiagnosis was also used in experiment 2, that had only three treated animals. In experiment 1, mRNA of $B$. burgdorferi could be detected in 3 of the 12 antibiotic-treated NHPs at post-mortem examination; however, borrelial DNA was not detected by PCR in any of these 3 animals at the same tissue site. Also, for 2 of the mRNA-positive NHPs, borrelial DNA could not be detected in any of the tissues examined. These are rather surprisingand paradoxical-results. It should be emphasized that many of these assays are presumptive rather than confirmatory for determining the viability of microorganisms; for example, neither the detection of DNA or mRNA is a definitive indicator of viability (Barer and Harwood 1999; Bogosian and Bourneuf 2001). Studies indicate that the likelihood of detecting mRNA after cell death in bacteria is related to the environmental conditions present, the method by which the microorganism was killed, the physiological state of the microorganism before killing, and the size and the region of the RNA transcript targeted (Sheridan et al. 1999; Birch et al. 2001; Reiman et al. 2010); mRNA can remain

\footnotetext{
${ }^{1}$ Division of Infectious Diseases, Department of Medicine, New York Medical College, Valhalla, New York.

${ }^{2}$ American Lyme Disease Foundation, Lyme, Connecticut.

${ }^{3}$ Lyme Borreliosis Unit, Health Protection Agency, Public Health Laboratory, Southampton General Hospital, United Kingdom.

${ }^{4}$ Department of Neurology and Neuroscience, University of Medicine and Dentistry, New Jersey-New Jersey Medical School, Newark, New Jersey.

${ }^{5}$ Department of Microbiology and Immunology, New York Medical College, Valhalla, New York.

${ }^{6}$ Departments of Pediatrics, Epidemiology, and Public Health, and of Investigative Medicine, Yale University, New Haven, Connecticut.
} 
undamaged for long periods if bacterial cells are destroyed by methods that inactivate RNase, rather than mRNA itself (Sung et al. 2004). Following exposure to potentially lethal methods (such as antibiotics, chemical agents, or heat), microorganisms are sometimes better characterized as moribund, rather than alive or dead.

In experiment 1 , when the antibiotic-treated and shamtreated groups were combined, a total of 17 NHPs were designated as persistently infected, with no difference in the rate of persistence between antibiotic-treated and sham-treated NHPs (Embers et al. 2012). In 11 (64.7\%) of the 17 NHPs, the sole basis for this assessment was a positive IFA, a procedure which in tissues is highly subjective and poorly reproducible. IFA is not a well-accepted assay in disseminated Lyme disease except in instances when antigen density is very high, such as in skin during early infection (Pachner et al. 2001). Given the very low antigen density expected in the animals studied by Embers and colleagues (2012), there is a high likelihood of false-positive results. It is therefore essential for individuals who perform these assays to be blinded to treatment group. If this was done, it was not mentioned in the reported study (Embers et al. 2012).

One treated animal and one untreated animal in experiment 1 were stated to be culture-positive. For some of the "culture-positive" animals the spirochete could not be subcultured, at least in experiment 2 . Since strains of B. burgdorferi that readily grow in vitro, such as the B31 strain, were used to infect the NHPs, this raises questions about whether the identified spirochetal forms were in fact viable. Although the specificity of culture is not $100 \%$ (Fallon et al. 2008), we agree that culture-positive animals who received antibiotics had convincing evidence of residual viable $B$. burgdorferi. Based on the results of treatment of $B$. burgdorferi infection in other reported animal models, this suggests that some or all of the animals were undertreated (Wormser et al. 2006; Wormser and Schwartz 2009). This could have been due to either an inadequate dosage or frequency of administration of the antibiotics. Alternatively, inadequate antibiotic exposure might have occurred in only a subset of the animals, due to variability in blood levels of drug among animals that received identical doses (Wormser and Schwartz 2009). This is not uncommon in outbred animals; in a study that measured levels of orally administered doxycycline in the blood of rhesus macaques, there was substantial variability in drug levels between animals (Kelly et al. 1992). Therefore, it is essential that antibiotic blood levels be closely monitored in every animal throughout the treatment period, particularly in a study with so few animals; this was not done by the Embers group (2012).

To our knowledge, pharmacokinetic (PK) data for doxycycline have never been determined in rhesus macaques. PK data for ceftriaxone have been determined for only seven rhesus macaques; none was noted to be of Chinese origin. The terminal half-life of ceftriaxone was quite variable, ranging from $89 \mathrm{~min}$ to $6.4 \mathrm{~h}$ (Matsui et al. 1984; Pelak et al. 1987; Sundelof et al. 1997). The usually reported half-life in adult humans is $6-8 \mathrm{~h}$ (Patel and Kaplan 1984). Given the high degree of variability of the half-life in macaques, it is certainly not justifiable for Embers and associates (2012) to assume that the dosage of ceftriaxone administered was comparable to that in humans given this antibiotic. Furthermore, until an erratum was posted recently (www.plosone.org/article/info\%3Adoi\%2F10.137\%2F journal.pone.0029914), the authors stated that they actually treated their animals with ceftiofur, not ceftriaxone; the PK of ceftiofur apparently has never been studied in any primate. Given the paucity of reported data on the PK of doxycycline, ceftiofur, and ceftriaxone in NHPs, there is no evidence to support the claim by the Embers group (2012), that one of the two primary reasons for using rhesus macaques was "the similarity between the available pharmacokinetics data for ceftriaxone and doxycycline in rhesus macaques and in humans."

The few doxycycline levels that were reported by Embers and associates (2012) were based on bioassays. Ideally, levels of doxycycline in blood should not be determined by bioassay, because such assays measure native drug plus any other active metabolites or chemical moieties. This can be problematic unless the bioassay incorporates B. burgdorferi, which was not the case in the bioassays of the Embers group (2012).

Without PK data or detailed information on the blood levels of the antibiotics administered at multiple time points, with their level of protein binding, it is impossible to ascertain the customary pharmacodynamic (PD) parameters for the antibiotics used in the experiments of Embers and co-workers (2012). With other infections, the PD parameter that seems to be most pertinent for the antimicrobial efficacy of doxycycline is the area under the concentration time curve at $24 \mathrm{~h}$ (AUC) of free drug divided by the minimum inhibitory concentration (MIC) of the infecting microorganism (Ambrose et al., 2007). For ceftriaxone, the PD parameter that correlates best with antibiotic efficacy is the duration of time that free drug in serum exceeds the MIC of the microorganism being treated (Ambrose et al. 2007).

It is also important to note that the Embers group (2012) used an extremely large dose of needle-inoculated spirochetes $\left(3.2 \times 10^{8}\right)$, injected in different sites, to produce a disseminated $B$. burgdorferi infection. The authors acknowledge that this in no way approximates a naturally-acquired infection; obviously under such circumstances extrapolation of the results obtained to naturally-acquired human disease is not valid.

There are other concerns regarding the extrapolation of the findings of the Embers group (2012) to humans. Most importantly, their findings in NHPs are not consistent with the results of human studies. Despite extensive testing by various diagnostic modalities including culture and PCR, there has been no evidence for the persistence of B. burgdorferi in treated patients in the United States, including patients with antibiotic-refractory arthritis or with post-treatment Lyme disease syndrome (PTLDS; Klempner et al. 2001; Klempner, 2002; Fallon et al. 2008; Li et al. 2011). The term PTLDS refers to patients with persistent subjective symptoms despite treatment with antibiotics and resolution of the objective manifestations of Lyme disease (e.g., erythema migrans; Wormser et al. 2006). In the absence of tissue inflammation or exotoxin production, it is also unclear how "persisters" might cause symptoms associated with Lyme disease or with PTLDS; indeed, the hypothesis that they would is counterintuitive for B. burgdorferi, as well as for other microorganisms. For example, only a tiny fraction of patients with acute mononucleosis develop persistent fatigue, but essentially all patients remain infected with the Epstein-Barr virus. Moreover, one-third of the world's 
population has persistent Mycobacterium tuberculosis infection without any symptoms. When latent tuberculosis reactivates there are clearly demonstrable objective findings. Long-term follow-up of Lyme disease patients with posttreatment subjective complaints indicates that these patients never manifest objective clinical manifestations such as arthritis (the most common late manifestation of Lyme disease in humans) at later time points (Nowakowski et al. 2003; Wormser et al. 2006). Thus, even when present, persistent microorganisms are largely irrelevant clinically unless they can be shown to cause disease.

A study of a Chinese rhesus macaque model for the evaluation of a recombinant OspA vaccine preparation for prevention of tick-transmitted B. burgdorferi infection may be instructive (Philipp et al. 1997). This study used some of the same assessment measures that the Embers group (2012) used, and was conducted by some of the same investigators. In this study, at least 9 of the 12 macaques that were vaccinated and later challenged by tick bite had molecular evidence ( 7 of 12 were PCR-positive), or had immunohistologic evidence of B. burgdorferi infection; curiously, the animals did not develop serologic evidence of infection and were culture-negative. Philipp and associates (1997) suggested that "these monkeys may have undergone a low level of infection that was transient." Although the findings could have been interpreted to suggest that the OspA vaccine was completely ineffective if one employs the criteria used by Embers and associates (2012) in the treatment study under discussion, clinical trials demonstrated just the opposite. The vaccine was found to be efficacious for humans, and the vaccine was approved by the FDA. Thus, as with any animal model, caution should be exercised in making extrapolations from the rhesus model to human disease.

In conclusion, it is not justifiable to assume that reliable information about human Lyme disease or PTLDS can be inferred from the methodologically flawed study of Embers and colleagues (2012) on the use of ceftriaxone and/or doxycycline for the treatment of latent $B$. burgdorferi infection in rhesus macaques. It is essential that future studies on the treatment of animals infected with B. burgdorferi address standard PK/PD parameters, and provide sufficient documentation of the consistency of antibiotic blood levels over time and among different animals.

\section{Author Disclosure Statement}

G.P.W. received research grants from the Centers for Disease Control and Prevention (CDC); the National Institutes of Health (NIH); Immunetics, Inc.; BioRad; DiaSorin, Inc.; and BioMerieux. He has equity in Abbott (which has no FDA-approved Lyme product to our knowledge). He is an expert witness in malpractice cases involving Lyme disease, is an unpaid board member the American Lyme Disease Foundation, and is an expert witness regarding Lyme disease in a disciplinary action for the Missouri Board of Registration for the Healing Arts. E.D.S. has served as an expert witness in legal proceedings related to the correct and incorrect diagnosis of Lyme disease, and is an unpaid board member of the American Lyme Disease Foundation. I.S. receives grants from the NIH and CDC. S.O'C. has served as an expert witness at a General Medical Council Fitness to
Practice hearing in relation to Lyme disease. No conflicts exist for the other authors.

\section{References}

Ambrose PG, Bhavnani SM, Rubino CM, et al. Pharmacokinetics-pharmacodynamics of antimicrobial therapy: it's not just for mice anymore. Clin Infect Dis 2007; 44:79-86.

Barer MR, Harwood CR. Bacterial viability and culturability. Adv Microbial Physiol 1999; 41:94-137.

Birch L, Dawson CE, Keer JT. A comparison of nucleic acid amplification techniques for the assessment of bacterial viability. Lett Appl Microbiol 2001; 33:296-301.

Bogosian G, Bourneuf EV. A matter of bacterial life and death. EMBO Reports 2001; 2:770-774.

Embers ME, Barthold SW, Borda JT, et al. Persistence of Borrelia burgdorferi in rhesus macaques following antibiotic treatment of disseminated infection. PLoS One 2012; 7(1):e29914; doi:10.1371/Journal.pone.0029914.

Fallon BA, Keilp JG, Corbera KM, et al. A randomized, placebocontrolled trial of repeated IV antibiotic therapy for Lyme encephalopathy. Neurology 2008; 70:992-1003.

Kelly DJ, Chulay JD, Mikesell P, et al. Serum concentration of penicillin, doxycycline, and ciprofloxacin during prolonged therapy in rhesus monkeys. J Infect Dis 1992; 166: 1184-1187.

Klempner MS. Controlled trials of antibiotic treatment in patients with post-treatment chronic Lyme disease. Vector Borne Zoonotic Dis 2002; 2:255-263.

Klempner MS, Hu LT, Evans J, et al. Two controlled trials of antibiotic treatment in patients with persistent symptoms and a history of Lyme disease. N Engl J Med 2001; 345:85-92.

Li X, McHugh G, Damle N, et al. Burden and viability of Borrelia burgdorferi in skin or joints of patients with erythema migrans or Lyme arthritis. Arthritis Rheum 2011; 63:2238-2247.

Matsui H, Kumiya M, Ikeda C, et al. Comparative pharmacokinetics of YM-13115, ceftriaxone, and ceftazidime in rats, dogs, and rhesus monkeys. Antimicrob Agents Chemother 1984; 26:204-207.

Nowakowski J, Nadelman RB, Sell R, et al. Long-term follow-up of patients with culture-confirmed Lyme disease. Am J Med 2003; 115:911-916.

Pachner AR, Ameniya K, Bartlett M, et al. Lyme borreliosis in rhesus macaques: effects of corticosteroids on spirochetal load and isotype switching of anti-Borrelia burgdorferi antibody. Clin Diagn Lab Immunol 2001; 8:225-232.

Patel IH, Kaplan SA. Pharmacokinetic profile of ceftriaxone in man. Am J Med 1984; 77:17-25.

Pelak BA, Gilfillan EC, Weissberger B, et al. Quaternary heterocyclylamino beta-lactams. VI. In vitro and in vivo antibacterial properties of L-642,946 and L-652,813, a long acting cephem. J Antibiotics 1987; 40:354-362.

Philipp MT, Lobet Y, Bohm RP Jr, et al. The outer surface protein A (OspA) vaccine against Lyme disease: efficacy in the rhesus monkey. Vaccine 1997; 15:1872-1887.

Reiman S, Grattepanche F, Rezzonico E, et al. Development of a real-time RT-PCR method for enumeration of viable $B i$ fidbacterium longum. Food Microbiol 2010; 27:236-242,

Sheridan GE, Szabo EA, Macket BM. Effect of post-treatment holding conditions on detection of tufA mRNA in ethanoltreated Escherichia coli: implications for RT-PCR-based indirect viability tests. Lett. Appl. Microbiol. 1999; 29:375-379.

Sundelof JG, Hajdu R, Gill CJ, et al. Pharmacokinetics of L749,345 , a long-acting carbapenem antibiotic, in primates. Antimicrob Agents Chemother 1997; 41:1743-1748. 
Sung KD, Stern NJ, Hiett KL. Relationship of messenger RNA reverse transcriptase-polymerase chain reaction signal to Campylobacter spp. Avian Dis 2004; 48:254-262.

Wormser GP, Dattwyler RJ, Shapiro ED, et al. The clinical assessment, treatment, and prevention of Lyme disease, human granulocytic anaplasmosis, and babesiosis: Clinical practice guidelines by the Infectious Diseases Society of America. Clin Infect Dis 2006; 43:1089-1134.

Wormser GP, Schwartz I. Antibiotic treatment of animals infected with Borrelia burgdorferi. Clin Microbiol Rev 2009; 22:387-395.
Address correspondence to: Gary P. Wormser

Division of Infectious Diseases Department of Medicine New York Medical College 40 Sunshine Cottage Road Valhalla, NY 10595

E-mail: gary_wormser@nymc.edu 